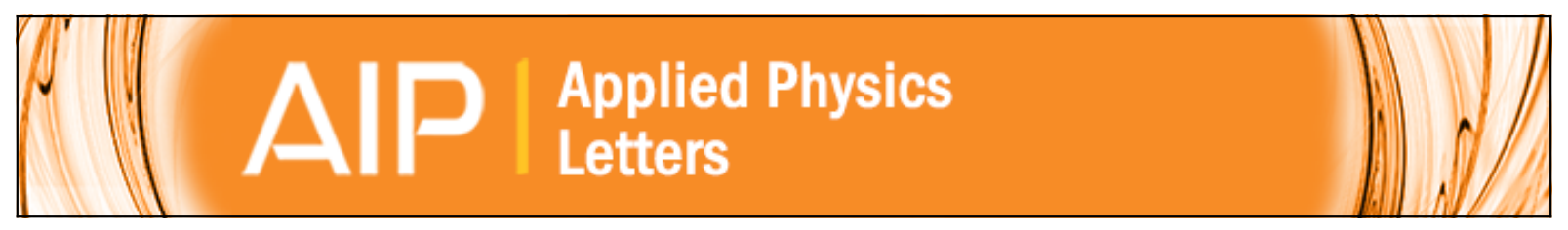

\title{
Resonance acoustic field position sensor
}

Shuxiang Dong, Feiming Bai, Jie-Fang Li, and Dwight Viehland

Citation: Applied Physics Letters 82, 4181 (2003); doi: 10.1063/1.1582355

View online: http://dx.doi.org/10.1063/1.1582355

View Table of Contents: http://scitation.aip.org/content/aip/journal/apl/82/23?ver=pdfcov

Published by the AIP Publishing

\section{Over 700 papers \& presentations on multiphysics simulation}

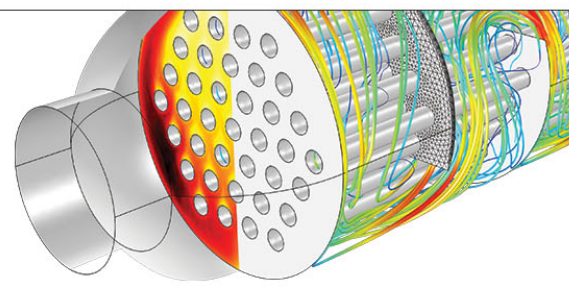




\title{
Resonance acoustic field position sensor
}

\author{
Shuxiang Dong, ${ }^{\text {a) }}$ Feiming Bai, Jie-Fang Li, and Dwight Viehland \\ Materials Science and Engineering, Virginia Tech, Blacksburg, Virginia 24061
}

(Received 13 March 2003; accepted 15 April 2003)

\begin{abstract}
An acoustic position sensor, based on a piezoelectric-sound-resonance cavity (PSRC), is reported in which a resonance acoustic field is used as the sensing mechanism. It has been discovered that an inserted object or an object motion in the sound radiation field results in changes in the resonance state of the PSRC. Experiments have demonstrated a high position resolution in the axial direction, and also good sensitivity in the transverse. () 2003 American Institute of Physics.
\end{abstract}

[DOI: 10.1063/1.1582355]

Current noncontact acoustic or ultrasonic position/object detections are based on time-of-flight or time-delay estimation using (i) pulse mode ${ }^{1-11}$ and (ii) continuous-wave (cw) mode. ${ }^{12-15}$ In pulse mode, acoustic sonar sensors or arrays actively transmit and receive reflected acoustic waves (echoes) from objects. This technology measures the transit time of echoes, and estimates the object distance by assuming that the sound velocity in the propagation medium (air or water) is known. Standard room-temperature ultrasonic technology based on pulse mode in air can resolve a position within $\sim 0.02 \mathrm{~mm}$ over ranges exceeding $100 \mathrm{~mm}^{8}$ To obtain a higher position resolution, or to monitor a fast-changing object, normally a higher-frequency sharp pulse is required. In the cw mode, the time-delay information is obtained by measuring the phase shift with a pair of transmitter and a receiver ${ }^{12,15}$ or a multifrequency excitation and detection sensor system. ${ }^{13,14}$ Though other methods (i.e., inductive and optical) are available, the acoustic echo method remains important for position/object sensing in many circumstances, where other methods have proven difficult or cost ineffective. ${ }^{8,10}$

Here, we report the development of a high-resolution piezoelectric-sound-resonance cavity (PSRC) sensor for generation of resonance acoustic fields, and subsequent detection of resonance state or impedance changes caused by object insertion or motion. Figure 1 illustrates the concept of a PSRC sensor. A disk-type piezoelectric thin layer/metal laminate $(8 \mathrm{~mm}$ in diameter), that has a center opening $(1 \mathrm{~mm}$ in diameter), is placed at one end of a small cylindrical cavity ( $5 \mathrm{~mm}$ in diameter and $3 \mathrm{~mm}$ in height). A capacitance type minisound sensor is then placed at the other end of the cavity. The piezoelectric film (100 microns in thickness) produces a bending vibration under a small applied ac voltage signal $\left(0.1-1.0 V_{\text {rms }}\right)$, forcing the acoustic medium (air or water) in the cavity to vibrate in a sound resonance state. A resonance acoustic field forms in the cavity, which radiates out from a center opening in the piezoelectric/metal laminate disk, along the axial or $\hat{z}$ direction of the cylindrical cavity. The minisound sensor at the opposite end of the cavity monitors pressure oscillations inside the cavity. The minisound sensor produces a voltage signal $\left(V_{s}\right)$ and phase $(\theta)$ which are proportional to the acoustic intensity $(I)$.

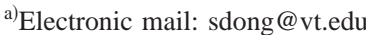

The PSRC sensing mechanism is based on the fact that when an object is placed near an acoustic wave radiation field, reflections are produced from the object. This disturbs the initial acoustic radiation field. The reflected wave also causes a change in the acoustic radiation impedance, including the radiation resistance $\Delta R_{r}$ and attachment radiation mass $\Delta M_{r}$. In some special positions $\left(N \lambda_{\text {air }} / 2, N=1,2, \ldots\right)$, the reflected waves interferes with the incident radiation wave, generating standing waves. Consequently, the acoustic resonance state (frequency and phase) of the cavity is altered. By using an acoustic sensor to monitor changes in the sound resonance field of the cavity, the static and dynamic positions of an object can be detected.

Suppose that the piezoelectric disk vibrates under an ac voltage $V_{E} \cdot{ }^{16}$ This forces the air in the cavity to contract and expand in a cyclic mode. The air vibrations in the cavity will then force the air column in the center opening to vibrate too, due to a pressure differential inside the cavity. We can use a model of a forced "mass-spring" system to describe the motion of the air column in the center-opening, in which the mass of the air column is $M_{M}$ and its spring constant is $K_{M}$. Treating the induced volume-velocity $U(=S d \xi / d t, S$ is the cross-sectional area of the center opening) and the pressure variable $p$ as a lumped parameter, ${ }^{17}$ according to Newton's second law, the solution to this equation of motion under an ac voltage $V_{E}$ is

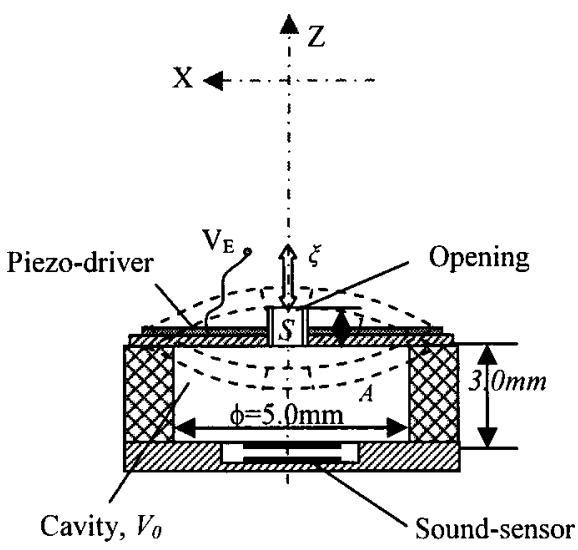

FIG. 1. Illustration of the concept of the PSRC sensor. A piezodriver is used for generating resonance sound field, and a sound sensor is used for monitoring the resonance acoustic field and its changes. 


$$
\begin{aligned}
U & =\frac{p}{Z_{A}} \\
& =-\alpha \frac{\rho_{0} c_{0}^{2} A}{2 V_{0}} \frac{V_{E}}{\left(R_{A}+R_{A r}\right)+j\left[\omega\left(M_{A}+M_{A r}\right)-1 / \omega C_{A}\right]},
\end{aligned}
$$

where $\alpha=e a^{2} h_{p} / 4(1+v) D_{\text {comp }}$ is a parameter related to the piezodriver, $c_{o}$ and $\rho_{o}$ are the air sound velocity and density, $Z_{A}$ is the acoustic impedance of the vibration system, $M_{A}$ $=M_{M} / S^{2}, R_{A}=R_{M} / S^{2}$, and $C_{A}=C_{M} S^{2}\left(C_{M}=1 / K_{M}\right)$ are the acoustic mass, acoustic resistance, and acoustic compliance of the air column in the center opening, respectively, and $M_{A r}=M_{r} / S^{2}$ and $R_{A r}=R_{r} / S^{2}$ are the acoustic attachment mass and acoustic radiation resistance, respectively. Following Eq. (1), the volume-velocity $U$ of the air flowing through the center opening is inversely proportional to $Z_{A}$. At the resonance state condition, $j\left[\omega\left(M_{A}+M_{A r}\right)-1 / \omega C_{A}\right]$ $=0$, the pressure oscillations inside the cavity are relatively large; consequently, the volume-velocity $U$ in the center opening is maximum.

In the free condition, the resonance frequency is $\omega_{0}$ $=1 /\left(M_{A} C_{A}\right)^{1 / 2}$. However, the cavity produces a strong radiation and a resonance sound field; thus, the resonance frequency is shifted by $M_{A r}$ to $\omega_{1}=1 /\left[\left(M_{A}+M_{A r}\right) C_{A}\right]^{1 / 2}$. The change in acoustic impedance (both $R_{A r}$ and $M_{A r}$ ) caused by the sound radiation will result in a change in $U$. A large acoustic impedance will result in a small $U$; consequently, a small $V_{s}$ will be generated in the minisound sensor of the cavity. On the contrary, a small acoustic impedance will result in a large $U$; consequently, a large $V_{s}$ will be generated. If the working frequency is kept unchanged, the acoustic impedance change will result in a phase shift, rather than a resonance frequency change.

Insertion of an object into the resonance sound radiation field, near the center opening of the cavity, will result in an acoustic impedance change due to reflection. Thus, by measurement of the sound signal change, we can detect the position of an object. Equation (1) is the primary equation which governs the operational principle of the PSRC position sensor. It provides a relationship between the volumevelocity $U$, applied voltage, and acoustical impedance changes due to object position and displacement.

The response of the PSRC sensor to a step-wise object movement was measured using a lock-in amplifier method. A brass disk with a diameter of $30.0 \mathrm{~mm}$ served as the object. This object was translated along the $\hat{x}$ axis, which was vertical to the $\hat{z}$ axis of the PSRC prototype. The prototype was mounted on a linear slider, which could be translated along the $\hat{z}$ axis with precision. The detected signals (both voltage and phase) from the minisound sensor in the PSRC were scanned and recorded by the lock-in amplifier, monitoring the change of the sound resonance state. The drive voltage was kept constant; thus, measured changes can be attributed to object position changes. Because the drive voltage and input current were small, the power consumption was $<1 \mathrm{~mW}$.

In order to determine the position resolution along the acoustic radiation direction, the object was fixed at a position of $15 \mathrm{~mm}$ in front of the center opening of the PSRC. Figures 2(a)-2(c) show the output voltage and phase for the PSRC prototype as a function of position for various step displace- (a) Step displacement: $\mathbf{4 0}$ micrometer

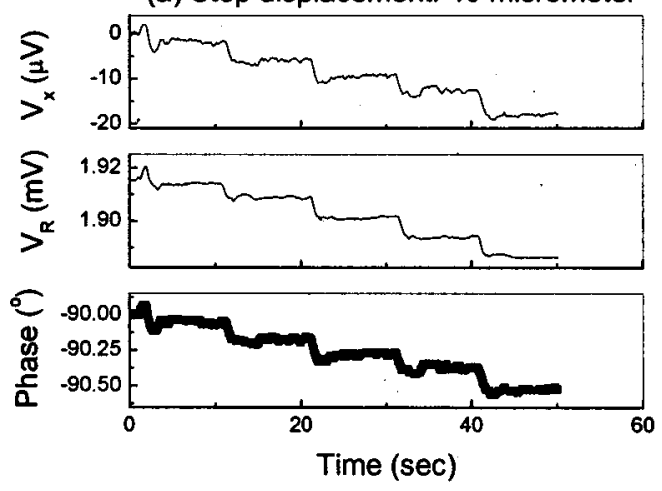

(b) Step displacement: -40 micrometer

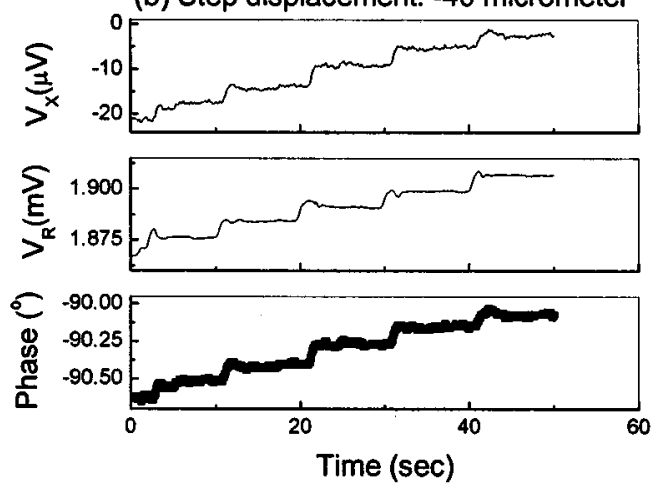

(c) Step displacement: 0-10 micrometer

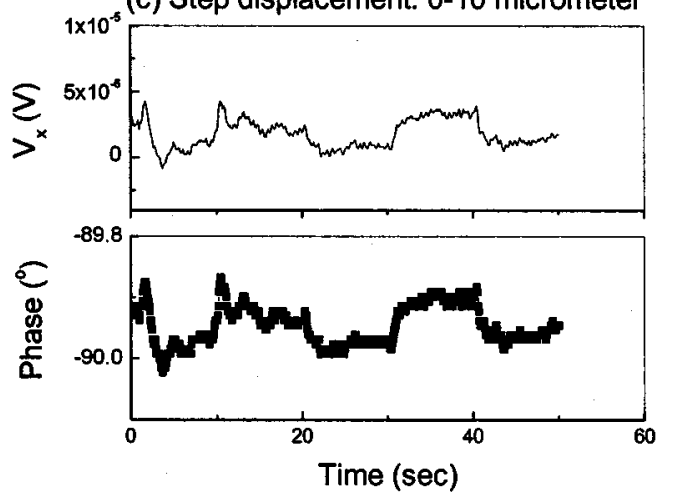

FIG. 2. Axial (longitudinal) position detection measurement (phase and voltage signal in PSRC) as a function of time for various step displacements of (a) $40 \mu \mathrm{m}$, (b) $-40 \mu \mathrm{m}$, and (c) $10 \mu \mathrm{m}$. The operational voltage and frequency were $0.9 V_{\text {rms }}$ and $11.699 \mathrm{kHz}\left(-90^{\circ}\right.$ phase state), respectively.

ments of $40 \mu \mathrm{m},-40 \mu \mathrm{m}$, and $10 \mu \mathrm{m}$, respectively. The time interval between step displacements was held constant at $10 \mathrm{~s}$ for all measurements. In Figs. 2(a) and 2(b), it can be seen that the PSRC prototype produces repeatable voltage and phase changes, in response to an object translation of either $\pm 40 \mu \mathrm{m}$. For smaller translations, the voltage and phase were both found to be linear functions of the object position, see Fig. 2(c). In Fig. 2, it can be seen that the minimum detectable displacement resolution is $<10 \mu \mathrm{m}$. For example, a $20 \mu \mathrm{m}$ position change resulted in a phase change $(\Delta \theta)$ of $\sim 0.12^{\circ}$ and a voltage change $\left(\Delta V_{x}\right)$ of $\sim 5 \mu \mathrm{V}$; and a $10 \mu \mathrm{m}$ position change resulted in $\Delta \theta$ $\sim 0.06^{\circ}$ and $\Delta V_{x} \sim 2.5 \mu \mathrm{V}$. The rates of phase and signal change to axial position translation along $\hat{z}$ were 6 $\times 10^{-3 \circ} / \mu \mathrm{m}$ and $0.25 \mu \mathrm{V} / \mu \mathrm{m}$, respectively.

These results clearly demonstrate that our PSRC proto- 


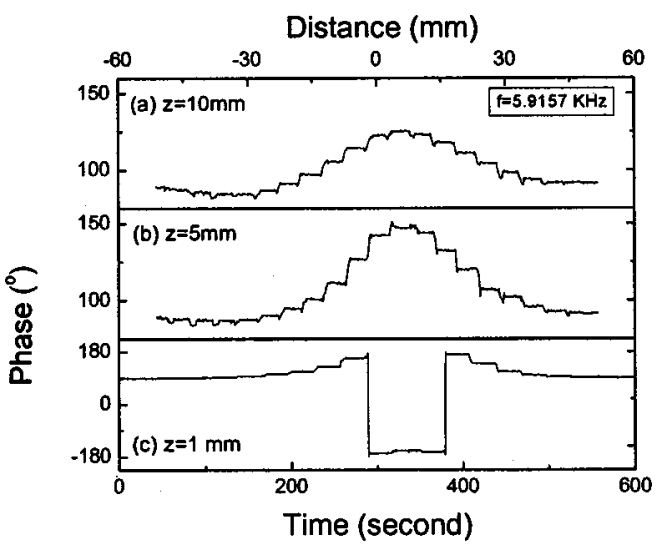

FIG. 3. Transverse position detection measurement. Phase signal in PSRC as a function of $x$-axis position and time for (a) $z=10 \mathrm{~mm}$, (b) $z=5 \mathrm{~mm}$, and (c) $z=1 \mathrm{~mm}$. The PSRC operational frequency was $5.92 \mathrm{kHz}\left(+90^{\circ}\right.$ phase state), and the drive voltage was $0.1 V_{\mathrm{rms}}$.

type has good axial resolution of small object displacements. The prototype has the capability of resolving changes in object position of $<10 \mu \mathrm{m}$, with an output voltage and phase that are linearly proportional to the position.

Larger-range axial displacements $(10<z<200 \mathrm{~mm})$ were also measured, in order to fully characterize the resolution capabilities of the position sensor of object detection. Measurements revealed that $V_{s}$ and $\theta$ are exponentially attenuated oscillation functions of the $\hat{z}$ axis position of the object. The exponential attenuation contains information that is a signature of the large axial range position change of the object.

Experimental measurements revealed that the PSRC is also sensitive to the transverse position and/or displacement of an object. In order to determine the transverse resolution along the $\hat{x}$ direction, which is vertical to the acoustic radiation field, the object was placed at different positions in front of the center opening of the PSRC and step wise translated from one side to another at step speed of $5 \mathrm{~mm}$ every $30 \mathrm{~s}$.

Figures 3(a)-3(c) show the sensor output voltage as a function of the transverse position at various positions of $z$ $=1,5$, and $10 \mathrm{~mm}$, respectively. It can be seen that the phase is dependent not only on the axial position of an object; but, also on transverse object displacements, over a distance of $\sim 100 \mathrm{~mm}$. A sensitive area of $\sim 60 \mathrm{~mm}$ (or $2 \times$ the diameter of the object) was found between $-22<x<38 \mathrm{~mm}$, where $\Delta \theta$ was a sinusoidallike function of position. The maximum phase change caused by object displacement (from center to side positions) was $\sim 36^{\circ}$ for $z=10 \mathrm{~mm}$ and $57^{\circ}$ for $z$ $=5 \mathrm{~mm}$. The average value of $\delta \theta / \delta x$ was $1.2 \times 10^{-3 \circ} / \mu \mathrm{m}$ for $z=10 \mathrm{~mm}$ and $1.9 \times 10^{-3 \circ} / \mu \mathrm{m}$ for $z=5 \mathrm{~mm}$.

When the object was placed close to the PSRC $(z$ $\leqslant 1 \mathrm{~mm}$ ), the phase changed from $\sim 90^{\circ}$ to $\sim 180^{\circ}$, as the object was translated from side to center. Near the center location, the phase rapidly dropped to $-180^{\circ}$ and remained constant in the vicinity of the center opening $(2<x$ $<14 \mathrm{~mm}$ ). These results demonstrate that a standing wave is formed when the object is close to the center opening. Near this position, reflection is so strong that the echo and incident radiation waves interfere with each other, resulting in a standing wave between the object and PSRC cavity bottom.

The results of this section demonstrate that our prototype PSRC sensor has good sensitivity to small transverse displacements. The transverse position resolution is $<100 \mu \mathrm{m}$. Over larger position ranges, the signal outputs (phase and voltage) are sinusoidal-like functions of position; but over small ranges they are linearly proportional to the object position.

Our resonance acoustic field position sensor is significantly different from conventional time-of-flight method, in which an ultrasonic transducer sends a very sharp pulse toward an object and measures the echo return time, or cwbased time-delay estimation method. In addition, the PSRC prototype offers the potential of accurate noncontact object detection in either gaseous or fluidic compressible media.

In summary, we have demonstrated a concept in position sensing which uses a PSRC sensing element. It is a simple, compact, robust, light weight sensor with good position sensitivity. It has very low working voltages $(<1 \mathrm{~V})$ and extremely low power consumptions $(<1 \mathrm{~mW})$. It offers a simple method to detect inserted objects and their motion in a compressible media, such as air or water, with high precision.

The work was supported by the Office of Naval Research.

${ }^{1}$ V. Magori, Proceedings of the 1994 IEEE Ultrasonic Symposium, 1994, p. 472.

${ }^{2}$ F. Ichikawa and T. Ohki, J. Acoust. Soc. Am. 90, 3384 (1991).

${ }^{3}$ G. Bucci and C. Landi, IEEE Trans. Instrum. Meas. 46, 1241 (1997).

${ }^{4}$ K. C. Eder and R. V. Krzyanowski, J. Acoust. Soc. Am. 83, 1710 (1988).

${ }^{5}$ C. M. Koukovinis, J. Acoust. Soc. Am. 80, 1277 (1986).

${ }^{6}$ R. M. White and S. Wenzel, J. Acoust. Soc. Am. 99, 2639 (1996).

${ }^{7}$ J. Yang, W.-S. Gan, and S.-E. Tan, Acoust. Resea. Lett. Online. 4, 47 (2003).

${ }^{8}$ D. A. Stubbs and R. E. Dutton, JOM 48, 29 (1996).

${ }^{9}$ A. Warszawski, Y. Rosenfeld, and I. Shohet, J. Comput. Civil Eng. 10, 67 (1996).

${ }^{10}$ K. Balasubramaniam, V. V. Shah, R. Daniel Costley, G. Bourdeaux, and J. P. Singh, Rev. Sci. Instrum. 70, 4618 (1999).

${ }^{11}$ H. Peremans, J. Acoust. Soc. Am. 102, 1567 (1997).

${ }^{12}$ J. C. Piquette and A. E. Paolero, J. Acoust. Soc. Am. 113, 1518 (2003).

${ }^{13}$ X. Li, E. Lasson, M. Sheplak, and J. Li, IEEE J. Ocean. Eng. 27, 47 (2002).

${ }^{14}$ X. Li, R. Wu, M. Sheplak, and J. Li, IEE Proc. F: Radar, Sonar, Naviga. 149, 53 (2002).

${ }^{15}$ C.-C. F. Tong and E. J. F. Barbieri, IEEE Trans. Instrum. Meas. 50, 1324 (2001).

${ }^{16}$ S. Dong, K. Uchino, L. Li, and D. Viehland (unpublished).

${ }^{17}$ A. D. Pierce, Acoustics (Acoust. Soc. Am, Woodbury, NY, 1989). 